\title{
Essais hydrauliques de l'inducteur hydrogène du moteur fusée Vulcain
}

\author{
R. Bernard et C. Rebattet CREMHyG Grenoble \\ J. Desclaux et F. Martignac SEP Vernon
}

\section{Introduction}

Une boucle d'essais de pompes à grande vitesse a été installée au Centre de recherches et d'essais de machines hydrauliques de Grenoble (CREMHyG) par la Société européenne de propulsion (SEP) qui est maître d'œuvre du moteur Vulcain du futur lanceur européen de satellites Ariane 5. Cette fusée, qui volera en 1995, est développée sous l'égide de l'Agence spatiale européenne (ESA), laquelle délègue au Centre national d'études spatiales (CNES) la direction technique et financière de ce programme.

A l'heure actuelle, le CREMHyG exécute sous contrat de la SEP des essais qui participent au développement de la turbopompe d'alimentation du moteur en hydrogène liquide. Cette machine, très puissante, et dont le rôle est essentiel, doit fournir un débit important d'hydrogène sous une pression élevée, tout en ayant une capacité d'aspiration à la plus basse pression d'entrée possible avec une conséquence directe sur l'indice constructif du lanceur, donc sur la capacité de charge utile. Les travaux en cours visent à la détermination des caractéristiques fonctionnelles de l'inducteur, ainsi qu'à l'étude des écoulements et de la cavitation.

L'intérêt des méthodes de simulation à partir de l'eau comme fluide d'essais est de simplifier grandement l'expérimentation qui peut être conduite avec plus de mesures et sur davantage de conditions de fonctionnement différentes. La quantité de résultats obtenus est plus importante qu'avec des essais en hydrogène liquide.

\section{Moyens d'essais}

\subsection{Boucle d'essais}

Cette boucle est implantée dans la plateforme d'essais CREMHyG (fig. l). Elle a été réalisée par Alsthom/ACB sous contrat SEP, le CREMHyG intervenant comme conseiller technique auprès de la SEP pour l'étude, la réalisation et la réception de l'installation. Pour des raisons de coût et d'encombrement, elle utilise une partie des équipements de la boucle principale d'essais de turbinespompes à laquelle elle est hydrauliquement raccordée. Sa mise en service a été effectuée en février 87. Elle permet de tester des pompes avec axe horizontal. Un entrainement à vitesse variable en continu permet des vitesses jusqu'à $8000 \mathrm{tr} / \mathrm{min}$ pour une puissance de $450 \mathrm{~kW}$. La tuyauterie est de diamètre $200 \mathrm{~mm}$ pour un débit de $200 \mathrm{l} / \mathrm{s}$ et une pression au refoulement de 40 bar.

La boucle a été équipée également d'un dispositif de génération et de mesures de germes de cavitation (brevet Neyrtec) permettant d'étudier l'influence des micro-bulles sur le déclenchement et le développement de certains types de cavitation. Le temps de transit de l'eau dans le circuit a été augmenté par l'adjonction d'un résorbeur permettant de fonctionner en injection de germes avec une concentration constante en amont de la pompe en essais. La teneur en air dissous est également contrôlée.

Toutes les grandeurs servant au calcul des performances globales sont acquises et traitées sur micro-ordinateur permettant, par la constitution de fichiers de points, d'ef-

\section{Hydraulic tests of the Vulcain rocket engine hydrogen inductor.}

Hydrodynamic tests of the prototype inductor of the Vulcain rocket engine hydrogen turbopump were conducted on the high speed section of the CREMHyG test bench in Grenoble. The test assembly, enginered by $S E P$, reproduces here the suction stage.

In addition to the usual characteristics (height, torque, efficiency and NPSH versus flowrate), the speed measurement campaign in the non-cavitating and cavitating running allows to specify the performances and to readjust the theoretical calculations. A visualization campaign has allowed for various cavitation figures to be identified; simultaneously, the measurements of peripheral pressures were recorded and they provide the fluid evolution along the hydraulic canal for several cavitation levels. 
fectuer des traitements graphiques et numériques «à la demande ". Les logiciels sont réalisés par le CREMHyG.

\subsection{Montage d'essais}

Le boitier (fig. 2) a été étudié de manière à pouvoir essayer l'étage inducteur de la turbopompe hydrogène du moteur Vulcain. L'étage inducteur se compose d'un inducteur double grille en tandem de diamètre $181,8 \mathrm{~mm}$ (une première grille à 4 pales d'angle moyen au bord d'attaque égal à $13,4^{\circ}$ et une deuxième grille flasquée à 12 aubes d'angle moyen $26,4^{\circ}$ ) et d'un redresseur à 13 aubes de diamètre de sortie égal à $149 \mathrm{~mm}$. Un disque d'équilibrage est installé sur le rotor pour la reprise des efforts axiaux engendrés par l'inducteur. Le montage est muni de deux manchettes (une pour la visualisation en altuglass, une en alliage d'aluminium pour les mesures).

Quatre types de mesures sont installés sur le montage (fig. 3) : pressions statiques pariétales en valeurs moyennes (55) et instantanées (8), vitesses (4), vibrations (5).

\subsection{Mesures}

\subsubsection{Mesures globales}

L'ensemble des performances globales sont réalisées par :

— un débitmètre à hélice de précision $5.10^{-3}$ de la mesure et de gamme $(0 ; 200) \mathrm{l} / \mathrm{s}$, redondé par un débitmètre électromagnétique ;

— un couplemètre à jauges sur l'arbre pompe de précision $3.10^{-3}$ de la mesure et de gamme $(0 ; 1000)$ N.m ;

- une roue phonique (vitesse de rotation) de précision

$10^{-4}$ de la mesure et de gamme $(0 ; 10000) \mathrm{tr} / \mathrm{min}$;

- des balances Desgranges et Huot (pressions moyennes) de précision $10^{-4}$ de la mesure, de gammes $(-1 ; 5)$, $(0 ; 30),(0 ; 60)$ bar relatif.

La précision obtenue est de 0,7 point sur la détermination du rendement (dont 0,5 point de répétabilité).

\subsubsection{Mesure de vitesse}

Pour ces mesures, il était nécessaire de disposer de sondes de petite taille perturbant peu l'écoulement, robustes afin de permettre des mesures en eau jusquà des vitesses de $40 \mathrm{~m} / \mathrm{s}$ dans un écoulement fortement turbulent, de fonctionnement éprouvé et de faible coût. Le choix s'est porté vers des sondes de type "Pitot" à 4 trous (fig. 4). Le CREMHyG dispose maintenant d'un anémomètre laser à rétrodiffusion.

Le CREMHyG a ainsi réalisé 5 sondes spécialement miniaturisées, permettant une mesure très fine de l'angle de l'écoulement dans le plan cylindrique et la détermination de l'angle dans le plan méridien, ainsi que la mesure du module de la vitesse moyenne.

Les sondes de vitesse sont reliées à des capteurs de pression de type Rosemount différentiels dont les gammes sont sélectionnées, soit pour l'équilibre des prises pseudo- statiques (mesure de l'angle), soit pour la mesure de la pression dynamique qui peut atteindre 8 bar.

A ce type de mesure, seront associées prochainement la vélocimétrie laser à fibre optique et les sondes à film chaud.

\subsubsection{Mesure de pression instantanée}

Les capteurs utilisés sont de type piézorésistifs. La vitesse de rotation importante du modèle en essais implique des mesures à des fréquences de plusieurs milliers de Hertz. Une centrale d'acquisition rapide permet des acquisitions simultanées dans les différents plans de mesures jusqu'à $300 \mathrm{kHz}$ (précision du convertisseur : 14 bits + signe). La mesure est acquise à une cadence de $20 \mathrm{kHz}$ soit $200 \mathrm{va}$ leurs par tour; deux catégories de mesures sont faites :

- une acquisition en continue sur 500 tours permettant d'obtenir un spectre de Fourier moyenné ;

- une série de 250 acquisitions successives d'une révolution d'arbre, le traitement donnant la moyenne de la pression et son écart-type calculés pour chaque $1 / 200^{\mathrm{c}}$ de tour sur les 250 tours.

Les conditions d'essais sont sévères : vibrations élevées à haut régime et cavitation. Le développement des moyens de mesures (capteurs et étalonnage dynamique) est une préoccupation importante du laboratoire.

\subsubsection{Moyens d'étalonnage et de traitement}

Les débitmètres sont étalonnés sur site avec une capacité jaugée de $60 \mathrm{~m}^{3}$. Des masses étalons permettent l'étalonnage du couplemètre et des balances de pression. Pour les sondes de vitesse, compte tenu du fort confinement de l'écoulement autour de ces dernières, un soin particulier a été porté à leur étalonnage en réalisant à cet effet une veine à section et vitesse variables reproduisant les dimensions de l'anneau liquide de l'inducteur. Les corrections à apporter dues à l'obstruction de la sonde ou les effets de la proximité des parois (fig. 5) peuvent être ainsi étudiées.

L'étude d'un banc d'étalonnage des capteurs de pressions instantanées en dynamique est en projet dans le cadre du groupe de travail $n^{\circ} 4$ de la SHF.

Divers micro-ordinateurs 16 et 32 bits permettent de gérer les différentes tâches d'acquisition rapide : pilotage de la boucle d'essais, analyse des signaux, étude spectrale, sorties graphiques... La compatibilité IBM-PC permet de s'interfacer avec un grand nombre d'instrumentation: carte d'acquisition, traitement d'image, laser...

\subsubsection{Visualisation}

En plus des prises de vues photographiques classiques, des films cinéma grande vitesse ont été réalisés pour obtenir des images instantanées des figures de cavitation, complétés par des prises de vues vidéo Umatic permettant d'obtenir des images moyennes de l'écoulement. Un système de traitement d'images, récemment acquis, devrait permettre de caractériser l'évolution des poches de cavitation. 


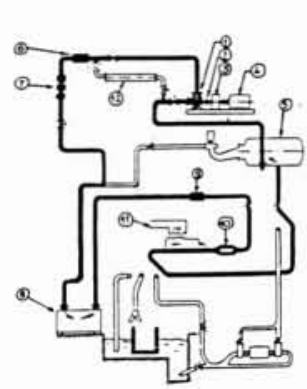

1. node1e

2.coupleatre

3. zultiplicateur

4. soteur

5.Cuve aspiration

6. Debitwètre helice

7. Monovars

8. Resorbeur

9. Deb.electromentetique

10.D1ssipateur

11. Echangeur

12. Veine d'ettalonnage

1. Schéma de la boucle d'essais.

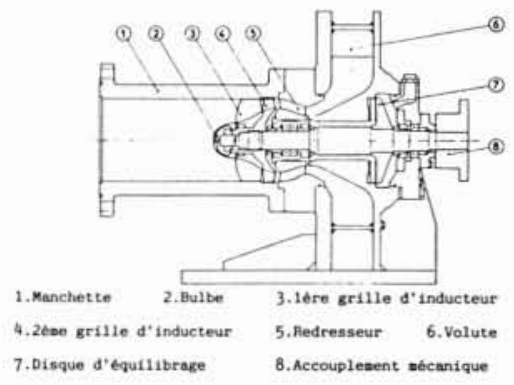

2. Schèma du montage d'essais d'inducteur.

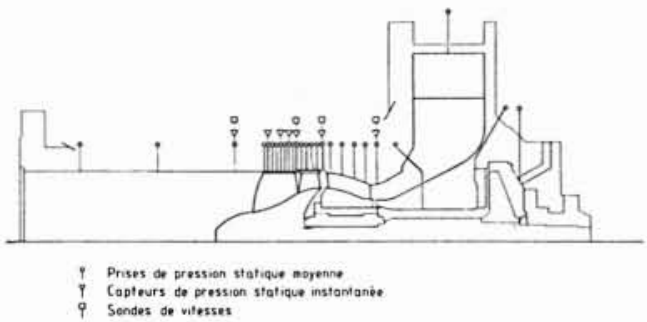

3. Implantation des mesures.

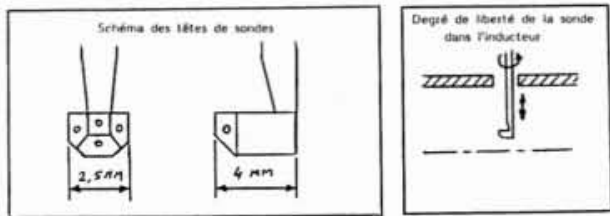

4. Sonde de vitesse

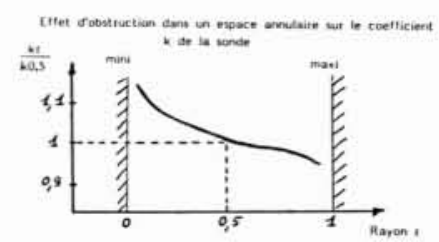

5. Exemple de coefficient d'étalonnage d'une sonde.

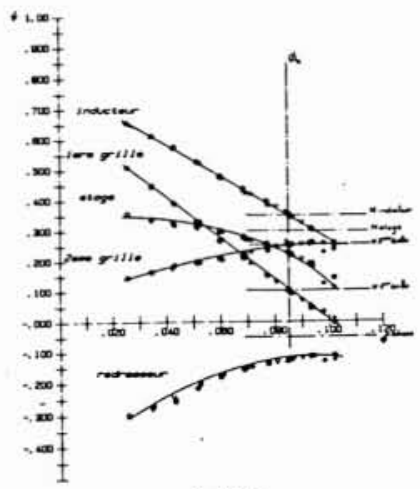

a. HMT

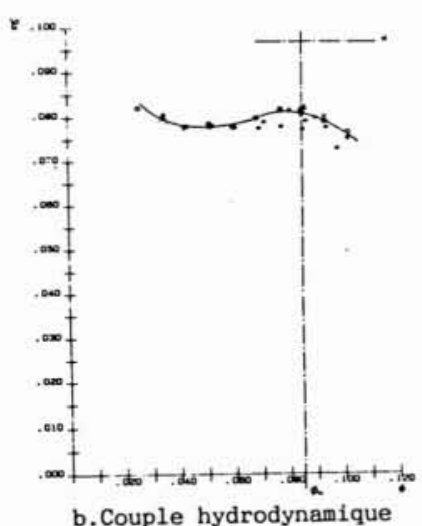

b. Couple hydrodynamique

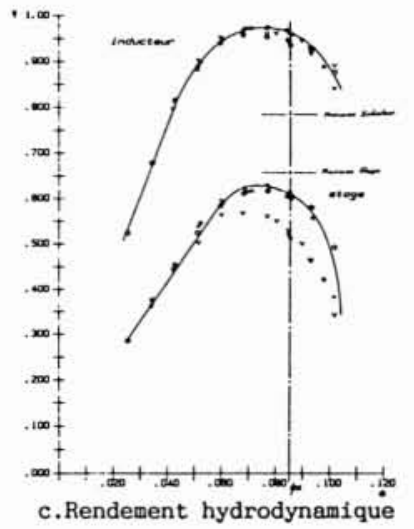

6. Caractẻristiques adimensionnelles.

\section{Résultats}

Les essais ont été menés en étroite collaboration entre les équipes SEP et CREMHyG.

\subsection{Caractérisation hydrodynamique}

Le relevé des pressions inter-éléments et du couple lors de la variation du débit entre 30 et $120 \%$ du débit nominal donne la figure 6 où sont représentées les caractéristiques en hauteur manométrique totale (HMT) des premières et deuxièmes grilles, de l'inducteur, du redresseur et de l'étage $(a)$ ainsi que celles du couple $(b)$ et du rendement hydrodynamiques $(c)$.
La caractéristique HMT de l'inducteur (de vitesse spécifique $N s=1,03 S I$ ) présente une quasi- linéarité de pente moyenne de 5,8 , inférieure à la pente de la caractéristique de la première grille égale à 6,8 , valeur typique de ce genre de grille d'aubes $(N s=2,52 S I)$. L'allure de la deuxième grille se rapproche d'une courbe spécifique d'une grille axiale $(N s=1,37 S I)$. Le redresseur provoque un niveau de pertes correct, mais son dimensionnement est susceptible d'amélioration. L'allure de la caractéristique en couple peut s'expliquer par la contribution prépondérante de la deuxième grille axiale à la consommation de couple de cet inducteur du type " high head". Les valeurs maximales de rendement atteintes sont .97 et .64 à $90 \%$ du débit nominal, un inducteur étant dimensionné en cavitation et non en p.m.r. 

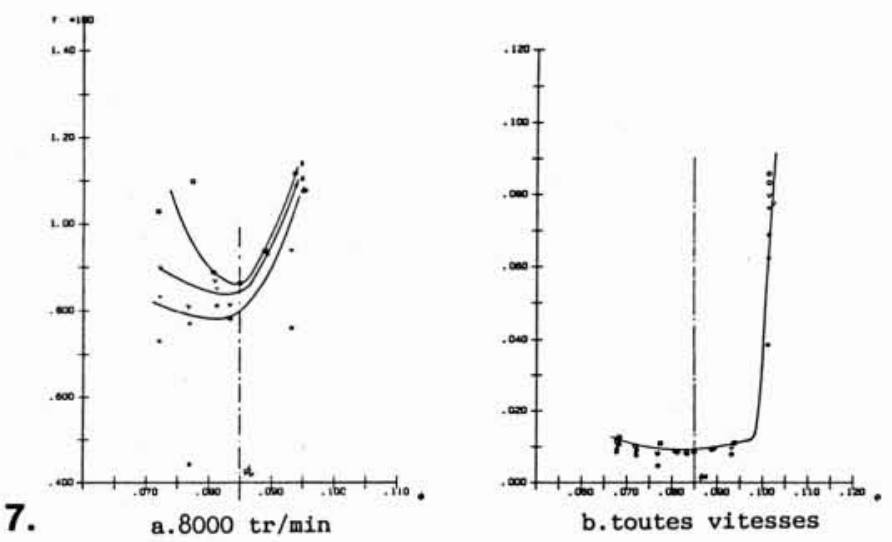

b. toutes vitesses

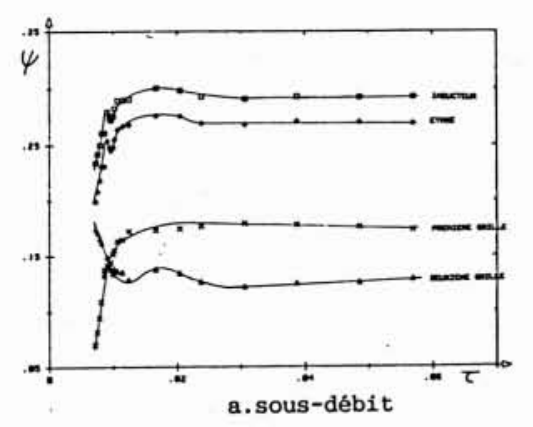

9.
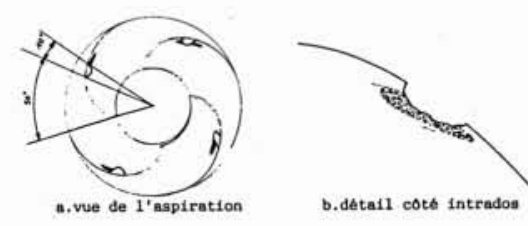

10.
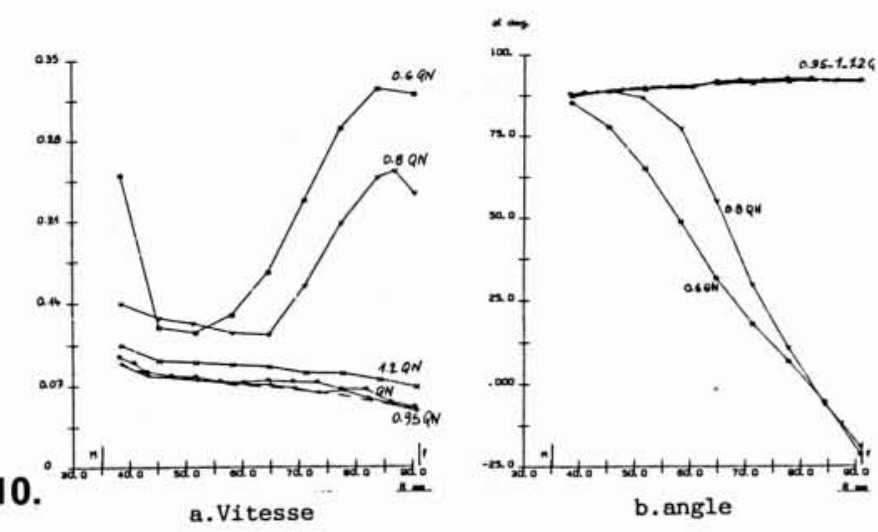

FLASQUB $=\frac{1}{5}$
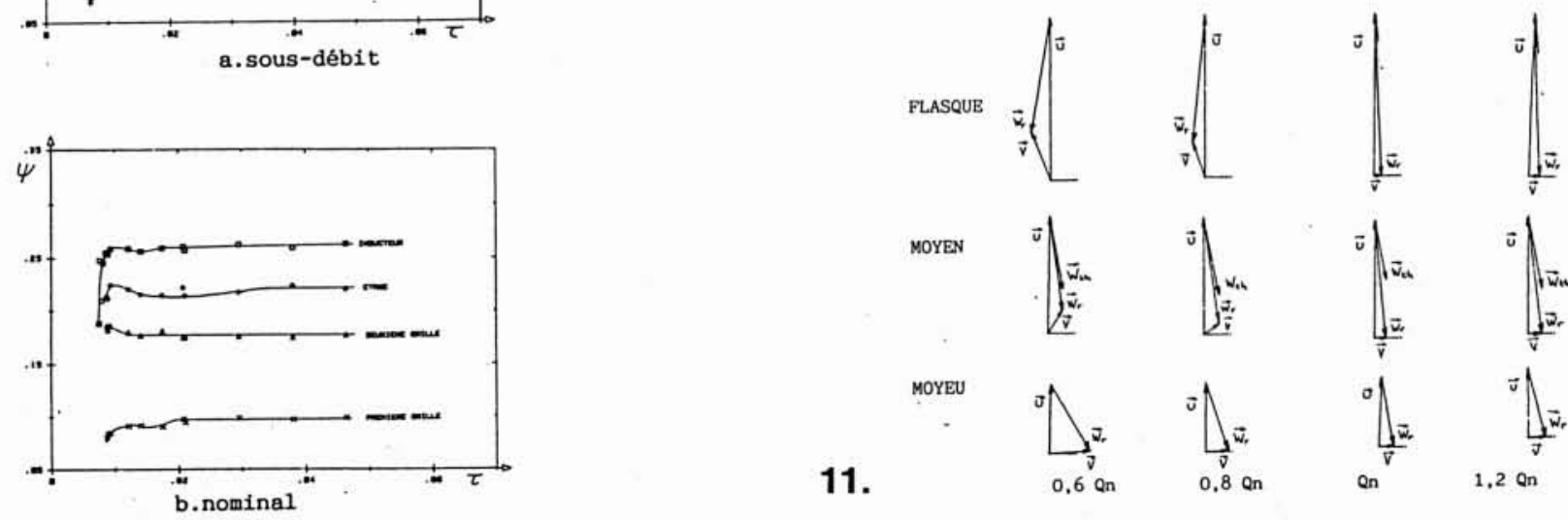

MOYEN
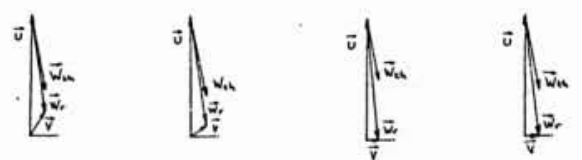

MOYEU
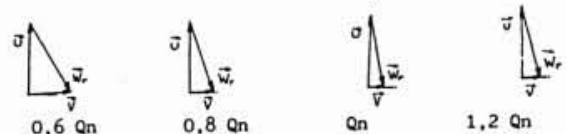

11.

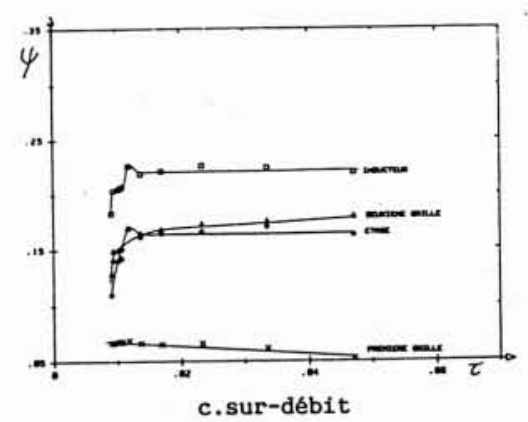

7. Caractéristique adimensionnelle en cavitation.

8. Influence du NPSH sur les hauteurs d'élévation statique des différents éléments.

9. Erosion du bord d'attaque de l'inducteur.

10. Sondage dans le plan amont de l'inducteur.

11. Triangles de vitesses à l'entrée de l'inducteur.

12. Sondage de vitesse en écoulement cavitant.
8.
. 


\subsection{Cavitation}

Le traitement des courbes d'influence du NPSH sur la hauteur manométrique statique de l'inducteur pour différents taux de chute de cette dernière $(3,5,10,20 \%)$ a abouti à la figure 7 ( $a: 8000 \mathrm{tr} / \mathrm{min}$ et $b: 4-6-8000 \mathrm{tr} / \mathrm{min})$ qui montre l'optimum au débit nominal et les remontées en sous et sur-débit. La très forte pente de la variation de NPSH en fonction du débit dénote la limitation de performances en cavitation de cet inducteur en dehors de son domaine d'utilisation et surtout de sa première grille en sur-débit.

Les comportements des deux grilles vis-à-vis de la cavitation est différente comme le montre la figure 8 .

En sous-débit $(a)$, le double décrochage de la courbe de l'inducteur est dû pour le premier, à la chute de la HMS de la première grille, et pour le second, la hauteur fournie par la $2^{\mathrm{e}}$ grille remonte la HMS de l'inducteur avant de décrocher à un NPSH plus faible. A $Q n(b)$, la $2^{\mathrm{c}}$ grille présente une courbe chutant pratiquement aux mêmes NPSH que la $1^{\text {re }}$ grille. En sur-débit $(c)$ la valeur quasi nulle de la HMS de la $\mathrm{I}^{\mathrm{re}}$ grille entraîne une alimentation constante de la $2^{\mathrm{e}}$ grille avec une valeur très faible de la pression d'où une cavitation rapide de cette dernière qui donne la chute de l'inducteur.

De plus, ces performances en cavitation peuvent être reliées à un état de cavitation par l'intermédiaire de la visualisation :

- cavitation d'entrefer : Elle est constituée de tourbillons de bulles dirigés vers l'aspiration, leur longueur varie suivant le point de fonctionnement. Ce type de cavitation est toujours présent à $8000 \mathrm{tr} / \mathrm{min}$;

- cavitation de bord d'attaque: Elle est caractérisée par une poche de vapeur accompagnée de filets de bulles accrochés à la génératrice du bord d'attaque côté extrados. L'apparition de ce type de cavitation survient à des NPSH assez faibles en commençant par la partie du bord d'attaque proche du moyeu ;

- cavitation de la deuxième grille tandem: La poche de cavitation apparait progressivement sur une puis deux pales dont le bord d'attaque est à proximité de chaque côté du bord de fuite de la grille précédente;

- fluctuations d'écoulement: Elles présentent un maximum lorsque les deux types de cavitation passent par intermittence le col. L'écoulement est relativement stable à fort NPSH (présence de la seule cavitation d'entrefer) et à très faibles NPSH (cavitation développée sur toute la $1^{\text {re }}$ grille).

L'érosion de cavitation a atteint deux types de matériel : les manchettes et la première grille. Il est apparu une dégradation importante de la manchette en altuglass et des parties dépolies sur celle en alliage d'aluminium. Cette érosion est essentiellement due au collapse des vortex issus de la cavitation d'entrefer. Une solution de réparation a été mise au point sur l'altuglass pour la suite des essais. De plus, lors d'un démontage, l'inducteur est apparu avec manque de matière sur chaque pale. Les quatre aubes présentent cet érosion au même point. A l'examen de l'environnement du trou, on peut supposer que le phénomène a été initié sur l'intrados (fig. 9).

On avait remarqué lors d'une maintenance de montage, la présence de traces dépolies au bord d'attaque côté intrados aux points décrits ci-dessus. L'érosion de cavitation avait commencé ses dégâts depuis le début des essais, et le phénomène d'incubation est démontré ici. L'incubation s'entend comme la non-linéarité dans le temps des effets visibles de l'érosion de cavitation. Il existe une première phase pendant laquelle on ne décèle aucune perte de masse mais la plupart des matériaux métalliques présentent des altérations locales tels que trous dont le nombre évolue avec le temps. Lorsque le vortex créé par la cavitation d'entrefer affleure la pale suivante, il rencontre une zone de compression du fluide à l'intrados. Dans cette zone, le collapse des bulles intervient et finit par attacher la matière. On peut attacher ce phénomène d'érosion à une durée d'essais en écoulement cavitant estimée à 75 heures.

\subsection{Vitesses d'écoulement}

Dans le but de connaître les champs de vitesse moyenne inter-éléments de l'inducteur, des sondages ont été effectués suivant quatre plans : entrée inducteur, inter-grille, sortie inducteur, sortie redresseur, à 3 débits (nominal $Q n$, $0,8 Q n, 1,2 Q n)$ et ceci pour des écoulements à différentes intensités de cavitation. Nous ne présentons ici que la mesure en amont de l'inducteur en non cavitant (fig. 10) ainsi que l'influence de la cavitation en sortie de la première grille au débit nominal (fig. 12).

Le phénomène apparaissant à l'entrée est caractéristique des inducteurs. Aux débits importants, supérieur à $0.8 Q n$, si l'écoulement est axial, par contre, aux débits faibles, une forte évolution du champ de vitesse apparaît du moyeu vers le flasque. Près du moyeu, si l'écoulement reste axial, la vitesse est grande comparée aux débits supérieurs. Lorsque le rayon de mesure s'éloigne de l'axe de rotation, l'angle absolu diminue et la direction de la vitesse se rapproche de la direction de la vitesse d'entraînement. L'angle méridien (vitesse par rapport à l'axe), augmentant aussi, il est évident que le fluide est entraîné en prérotation avec un effet centrifuge qui tend à augmenter la pression. Lorsque l'angle absolu s'annule vers le flasque, la vitesse étant maximale, le fluide n'est donc plus débitant. A un rayon supérieur, l'angle absolu devient négatif, l'inversion de la circulation du fluide montre que le fluide passe en périphérie de l'intrados à l'extrados de la pale. Ce champ de vitesse se retrouve sur les triangles de vitesses donnés en figure $1 /$ où la vitesse s'inverse en dessous du débit nominal au flasque. Au rayon moyen, la prérotation se fait encore sentir pour être sans effet au moyeu.

Pour trois états de cavitation en plus du non-cavitant, les répartitions radiales des modules de la vitesse et de l'angle absolu ainsi que de la pression totale ont été mesurées.

L'influence de la cavitation est prépondérante pour une chute importante de performances (10\%), l'effet étant plus sensible au moyeu qu'au flasque, la pression totale chutant de $60 \%$ vers le moyeu. 
13.

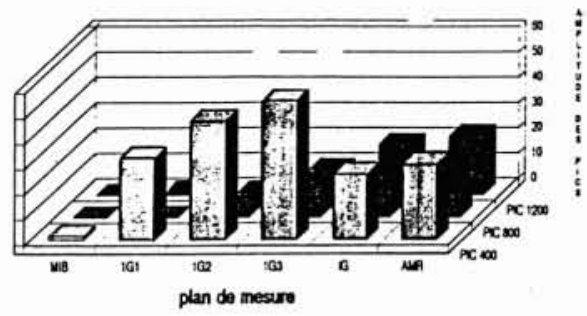

14.

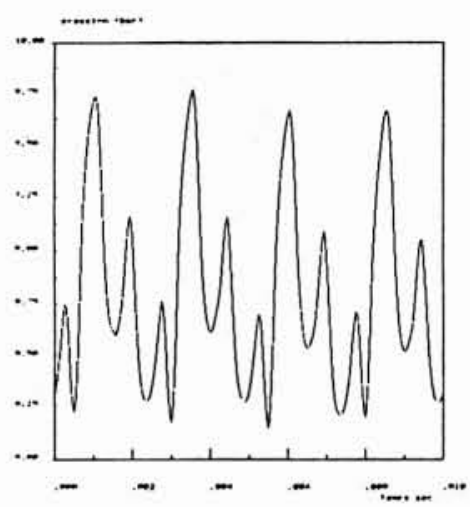

15.

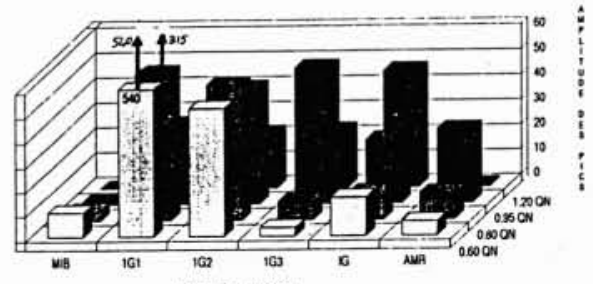

plan de mesure

13. Evolution des raies synchrones dans le modèle au débit nominal.

14. Signal temporel moyenné à l'intergrille au débit nominal.

15. Evolution de la raie $400 \mathrm{~Hz}$ dans le modèle en fonction du débit.

16. Spectres présentant des raies asynchrones.

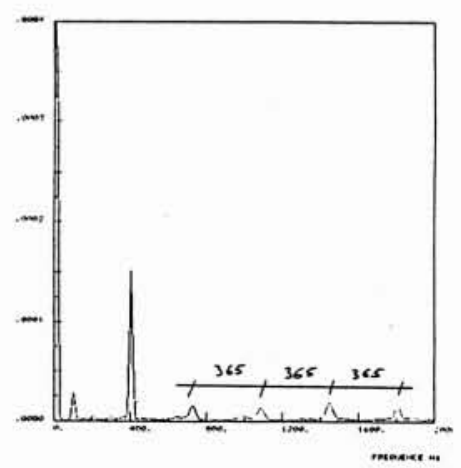

a.aspiration

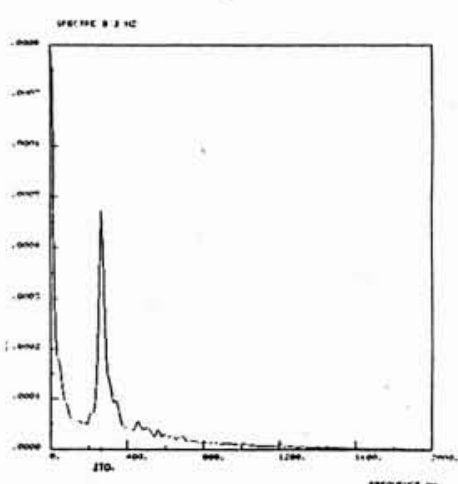

16.

b. refoulement

\subsection{Pressions statiques en valeurs instantanées}

On distingue deux types de fluctuations de pression qui ne sont pas discernés par les capteurs de pressions instantanées : celles dues à la turbulence et celles à caractère acoustique.

Les premières se propagent dans l'écoulement à une vitesse voisine de celui-ci et sont amorties rapidement. Les deuxièmes se propagent à la vitesse du son à travers le liquide sur de grandes distances car faiblement amorties.

Indépendamment de leurs types, on peut les classer en fonction du processus qui leur donne naissance :

- les mécanismes de génération à caractère aléatoire (turbulence, cavitation, ébullition) ;

- les mécanismes de génération à caractère périodique (vortex, pulsations de pression dues au mouvement des parties mobiles - rotation de l'arbre, passage ou coïncidence des aubes);

- les mécanismes d'amplification (résonances des volumes et des colonnes liquides donnant les fréquences caractéristiques du réseau vues par les capteurs amont et aval).

Les mesures ont été faites dans 6 plans (entrée, $1^{\text {re }}$ grille (3), amont et aval du redresseur) pour 4 débits (nominal $Q n, 0,6 Q n, 0,8 Q n, 1,2 Q n)$ à $6000 \mathrm{tr} / \mathrm{min}$.

L'examen des spectres fait apparaître deux types de raies, synchrones et asynchrones.

La figure 13 illustre l'évolution des raies synchrones 400 , 800 et $1200 \mathrm{~Hz}$ pour tous les plans au débit nominal. La vitesse de rotation est de $6000 \mathrm{tr} / \mathrm{min}$ soit une fréquence de passage des aubes de $400 \mathrm{~Hz}$ pour la première grille (4 pales) et $1200 \mathrm{~Hz}$ pour la deuxième grille (12 pales).

Le passage $1^{\text {re }}$ grille- $2^{\mathrm{C}}$ grille se voit parfaitement par le changement d'amplitude maximales entre les raies $400 \mathrm{~Hz}$ et $1200 \mathrm{~Hz}$. A l'intergrille, le signal temporel moyenné (fig. 14) montre la coexistence des deux raies.

L'influence du débit, donnée par la figure 15 (pic $400 \mathrm{~Hz}$ ), montre l'augmentation des fluctuations de pression en sous-débit à l'entrée de l'inducteur (prérotation) ainsi qu'en sur-débit à la sortie (entraînement).

Trois types de raies asynchrones ont été relevées sur les spectres dont une à $310 \mathrm{~Hz}$ inexpliquée à ce jour, peut-être une fréquence propre de pale. La deuxième raie asynchrone est constituée par une succession de pics espacés de $365 \mathrm{~Hz}$ au débit nominal (fig. 16a). La troisième raie asynchrone $(270 \mathrm{~Hz})$ est relevée sur le spectre linéaire à $0,60 Q n$ sur le plan aval redresseur (fig. 16b).

Sur la figure 16a, compte tenu de la géométrie de la ligne pour déterminer la célérité de propagation des ondes élastiques, le calcul de la période d'un aller-retour depuis le bulbe au premier coude nous permet de retrouver la fréquence de succession des pics qui est donc due aux différents modes de résonance de la colonne liquide amont. Sur la figure $16 b$, comme en amont, cette raie est liée à une résonance de boucle. Le refoulement se produit dans un divergent conique de longueur droite $1 \mathrm{~m}$. Si l'on suppose qu'à faible débit, le manque de circulation de l'eau constitue au niveau du coude un obstacle à la propagation d'onde élastique on peut alors comparer la portion droite en aval pompe à une cavité résonante du type d'Helmholtz. 
Effectivement, la formulation analytique des fréquences propres de résonateur de forme conique donne la valeur de cette raie. Ce mode est unique, il n'y a pas d'harmonique puisqu'il s'agit d'une résonance d'un système à un degré de liberté.

\section{Conclusion}

Les essais de performances de l'inducteur hydrogène à l'eau ont montré un bon fonctionnement de l'inducteur et un fonctionnement satisfaisant du redresseur. Les caractéristiques fonctionnelles ont été établies sur une large plage de coefficient de débit, et leur indépendance vis-à-vis des vitesses de rotation essayées a pu être vérifiée.

Les essais de cavitation ont montré que les valeurs de NSPH requis en chute de hauteur sont minimales au débit nominal avec performance de vitesse spécifique d'aspira- tion $N_{S A}=18,20(S I)$ très satisfaisante. De plus, les figures de cavitation ont pu être largement observées, ainsi que leur impact sur les vitesses d'écoulement. Des modèles existent pour transposer ces résultats en performances sur la machine réelle à pleine vitesse en hydrogène liquide, modèles empiriques présentant un degré d'imprécision sensible. Néanmoins, l'excellente performance acquise à l'eau permet de présager que l'objectif a des chances sérieuses d'être également atteint en fonctionnement sur le moteur-fusée.

L'érosion de cavitation a été constatée sur les pales de l'inducteur, et l'aspect agressif de certains régimes de cavitation observé pour une période cumulée relativement rapide ( 75 heures de cavitation sévère sur du titane).

Cette campagne d'essais fut également très enrichissante pour confronter les modèles théoriques de prévision développés par la SEP aux phénomènes réels, ainsi que pour appréhender le fonctionnement complexe de deux grilles d'aubes mobiles en tandem de forte puissance spécifique.

\author{
Messieurs R. Bernard et C. Rebattet \\ CREMHYG-ENSHMG \\ BP 95 \\ 38402 Saint-Martin-d'Hères \\ Tél. : (16) 76825195
}

Adresses des auteurs

Messieurs J. Desclaux et F. Martignac
SEP Vernon
BP 802
27207 Vernon
Tél.: (16) 32217200

M. LECOFFRE: Je voudrais poser une question concernant les aspects thermodynamiques. Vous parlez de résultats en hydrogène. Quelle méthode pensez-vous utiliser pour transposer les résultats obtenus? Pensez-vous le faire à partir de corrélations ou pensez-vous vous servir d'une procédure particulière partiellement basée sur le modèle de Billet?

M. MARTIGNAC: On utilise une méthode qui a été développée par des ingénieurs américains de la NASA. Nous allons essayer, au cours de ces essais, de travailler sur la méthode d'entraînement qui va déterminer le transfert de masse de chaleur au niveau des poches de cavitation. Il y a eu plusieurs publications à ce sujet et on pourrait en discuter pendant longtemps.

Le reste fait l'objet d'une recherche qui est menée avec le CREM-HyG concernant des modèles qui sont un peu moins empiriques et qui prennent davantage en compte les phénomènes physiques. Tout un programme a démarré là-dessus depuis 1985 .

M. DOREY: Vous avez fait des mesures de fluctuation de pression. Les avez-vous corrélées avec le NPSH $3 \%$ que vous avez observé?

M. MARTIGNAC: Pour certains problèmes de mesures présen- tées, nous avons eu des difficultés avec la résistance des capteurs. Nous fonctionnons dans des régimes avec de très fortes vibrations et les capteurs n'ont pas très bien tenu. De ce fait, on n'a pas pu faire de mesure, en cavitation pour l'instant, mais c'est en projet.

M. VERRY: Lors des essais que nous avons effectués, nous avons constaté des pulsations basse fréquence lorsque les poches atteignent le col d'entrée de l'hélice.

Lorsqu'on a des poches beaucoup plus longues, on retrouve la stabilité. Est-ce un problème sérieux pour vous sur le plan industriel?

M. DESCLAUX : Vis-à-vis du fonctionnement de la turbopompe, on a un certain nombre de points critiques dont la plupart sont liés aux phénomènes d'instationnarité, en particulier à la vitesse de $34000 \mathrm{tr} / \mathrm{min}$. Il est très important de connaître quels sont les effets asynchrones que l'on peut rencontrer sur l'inducteur. Nous essayons de mener une démarche où nous essayons de coupler les informations qui peuvent nous venir par les pressions instantanées et les informations qui peuvent nous venir de jauges de contrainte installées soit sur les aubes, soit sur l'arbre, ce qu'on envisage pour le futur. 